\title{
Implications of an R-Scan for Charm Physics *
}

\author{
Johann H. Kühn \\ Institut für Theoretische Teilchenphysik, Karlsruher Institut für Technologie (KIT) \\ 76128 Karlsruhe, Germany \\ Johann.Kuehn@KIT.edu
}

\begin{abstract}
The impact of improved measurements of the cross section for electron-positron annihilation into hadrons in the charm threshold region is discussed. Two aspects are studied in detail: i.) A significant reduction of the experimental error of the electronic width of the narrow resonances $J / \psi$ and $\psi^{\prime}$ and of the continuum cross section from the open charm threshold up to $4.6 \mathrm{GeV}$ will lead to a correspondingly improved determination of the charmed quark mass. ii) A high luminosity measurement with $24 \mathrm{pb}^{-1}$ at three points and with a spacing of $2 \mathrm{MeV}$ around $\sqrt{s}=3511 \mathrm{MeV}$ may allow to observe the direct, resonant production of $\chi_{c 1}$.
\end{abstract}

Keywords: Perturbative quantum chromodynamics, charmed quarks, charmonium

PACS numbers:12.38.Bx, 14.65.Dw, 14.65.Fy

\section{Introduction}

Significant improvements in luminosity are anticipated for electron positron collisions in the low energy region, with the BES III experiment at the BEPC II collider in Beijing as characterisic example. BEPC II will cover the energy region between 2 and $4.6 \mathrm{GeV}$ and is therefore specifically suited for the exploration of the charm system. Indeed, recently a lot of emphasis has been put on the study of the newly discovered charmonium resonances denoted X,Y and Z. (For discussions of recent theoretical and experimental developments see e.g. Refs. 1,2). In this brief contribution two different aspects of charm physics will be investigated which could profit from a high luminosity scan in the charm threshold region:

i) When combined with increasingly precise measurements of the electronic widths of $J / \psi$ and $\psi^{\prime}$ an R-scan with larger luminosity and reduced systematical error could lead to an improved determination of the charm quark mass.

ii.) Investing $24 \mathrm{pb}^{-1}$ of integrated luminosity into the R-measurement at three points each, with a spacing of $2 \mathrm{MeV}$ around $3.511 \mathrm{GeV}$, the direct resonant production of $\chi_{c 1}$ (and perhaps even $\chi_{c 2}$ ), which can proceed through two virtual photons, seems within reach.

*Presented at PHIPSI13 - International Workshop on e+e- collisions from Phi to Psi 2013, Rome, Italy, 9-12 September 2013. 
Table 1. Experimental moments in $(\mathrm{GeV})^{-2 n}$ as defined in Eq. (1), separated according to the contributions from the narrow resonances, the charm threshold region and the continuum region above $\sqrt{s}=4.8 \mathrm{GeV}$. In the last column the NLO contribution from the gluon condensate is shown.

\begin{tabular}{c|lll|l||l}
\hline$n$ & $\begin{array}{l}\mathcal{M}_{n}^{\text {res }} \\
\times 10^{(n-1)}\end{array}$ & $\begin{array}{l}\mathcal{M}_{n}^{\text {thresh }} \\
\times 10^{(n-1)}\end{array}$ & $\begin{array}{l}\mathcal{M}_{n}^{\text {cont }} \\
\times 10^{(n-1)}\end{array}$ & $\begin{array}{l}\mathcal{M}_{n}^{\text {exp }} \\
\times 10^{(n-1)}\end{array}$ & $\begin{array}{l}\mathcal{M}_{n}^{\text {np }}(\mathrm{NLO}) \\
\times 10^{(n-1)}\end{array}$ \\
\hline 1 & $0.1201(25)$ & $0.0318(15)$ & $0.0646(11)$ & $0.2166(31)$ & $-0.0002(5)$ \\
2 & $0.1176(25)$ & $0.0178(8)$ & $0.0144(3)$ & $0.1497(27)$ & $-0.0005(10)$ \\
3 & $0.1169(26)$ & $0.0101(5)$ & $0.0042(1)$ & $0.1312(27)$ & $-0.0008(16)$ \\
4 & $0.1177(27)$ & $0.0058(3)$ & $0.0014(0)$ & $0.1249(27)$ & $-0.0013(25)$ \\
\hline
\end{tabular}

Table 2. Results for $m_{c}(3 \mathrm{GeV})$ in $\mathrm{GeV}$. The errors are from experiment, $\alpha_{s}$, variation of $\mu$ and the gluon condensate.

\begin{tabular}{c|l|llll||c}
\hline$n$ & $m_{c}(3 \mathrm{GeV})$ & $\exp$ & $\alpha_{s}$ & $\mu$ & $\mathrm{np}_{\mathrm{NLO}}$ & total \\
\hline 1 & 0.986 & 0.009 & 0.009 & 0.002 & 0.001 & 0.013 \\
2 & 0.975 & 0.006 & 0.014 & 0.005 & 0.002 & 0.016 \\
3 & 0.975 & 0.005 & 0.015 & 0.007 & 0.003 & 0.017 \\
4 & 0.999 & 0.003 & 0.009 & 0.031 & 0.003 & 0.032 \\
\hline
\end{tabular}

\section{Quark mass determination in $\mathrm{N}^{3} \mathrm{LO}$}

As discussed in the literature ${ }^{3,4,5,6}$, moments of the charm production cross section $\sigma\left(e^{+} e^{-} \rightarrow c \bar{c}+X\right) \equiv R_{c} \sigma_{0}$ with $\sigma_{0} \equiv \frac{4 \pi}{3} \frac{\alpha^{2}}{s}$, allow for a fairly precise determination of the charm mass. The $n$-th moment of $R_{c}$, as defined through

$$
\mathcal{M}_{n}^{\exp } \equiv \int \frac{\mathrm{d} s}{s^{n+1}} R_{Q}(s)
$$

can be experimentally determined from analysis of $e^{+} e^{-}$data. The quark mass (defined in the $\overline{M S}$ scheme at renormalization scale $\mu$ ) is then obtained from

$$
m_{Q}(\mu)=\frac{1}{2}\left(\frac{9 Q_{Q}^{2} \bar{C}_{n}}{4 \mathcal{M}_{n}^{\exp }}\right)^{1 /(2 n)} .
$$

The coefficients $\bar{C}_{n}$ which are required for this analysis depend on $\mu$ and the strong coupling constant and have been evaluated in three- ${ }^{7,8}$, later in four-loop approximation $^{9,10,11,12,13}$. The corresponding NNLO and $\mathrm{N}^{3} \mathrm{LO}$ charm- and bottomquark-mass determinations have been performed in Ref. 3 and Refs. 4, 5, 6, respectively. The moments are conveniently split into three pieces, one from the narrow resonances $J / \psi$ and $\psi^{\prime}$, one from the threshold for open charm production up to $4.8 \mathrm{GeV}$ and one from the perturbative continuum above $4.8 \mathrm{GeV}$. Their respective contributions and the corresponding errors are shown in Table 1, the results for the quark mass $m_{c}(3 \mathrm{GeV})$ are given in Table 2 . A detailled discussion of the theory uncertainty can be found in Refs. 4,6 .

The path to further improvements is evident from Tables 1 and 2. The experimental input is dominated by the electronic width $\Gamma_{e}$ of the narrow resonances. Considering the enormous improvements in luminosity and experimental techniques during the past years, a reduction of the error on $\Gamma_{e}$ by a factor two seems feasible ${ }^{14}$. 
The planned scanning with drastically increased luminosity will, furthermore, allow for a similar reduction of the error in the threshold region ${ }^{15}$. At the same time a precise measurement at the highest accessible energies arount $4.6 \mathrm{GeV}$ and the comparison of the result with the prediction of perturbative QCD will strengthen the confidence in the applicability of pQCD at higher energies. Performing a combined fit for the lowest three moments, thus exploiting the information of the data in an optimal way, may lead to a further reduction of the experimental error. The reduction by a factor two of the error on $\mathcal{M}_{n}^{\exp }$ is thus well within reach.

The choice of the strong coupling constant $\alpha_{s}$, its central value as well as its error, is a matter of disput- ${ }^{16}$. In our analysis $\alpha_{s}\left(M_{Z}\right)=0.1189 \pm 0.0020$ has been adopted. The dependence of our result on $\alpha_{s}$ can be directly deduced from the final result

$$
m_{c}(3 \mathrm{GeV})=\left(986-\frac{\alpha_{s}-0.1189}{0.002} 9 \pm 10\right) \mathrm{MeV}
$$

which had been based on the second moment. Evolving, for example, to the scale of $m_{H}$, one obtains

$$
m_{c}(126 \mathrm{GeV})=\left(609-\frac{\alpha_{s}-0.0189}{2} 15 \pm 6\right) \mathrm{MeV}
$$

where the parametric $\alpha_{s}$-dependence affecting both the evaluation of $m_{c}(3 \mathrm{GeV})$ and its evolution from $\mu=3 \mathrm{GeV}$ to $126 \mathrm{GeV}$ are combined. This result is evidently a critical input for predictions of the Higgs boson decay rate into $c \bar{c}$ and implies an error of $5.3 \%$ from this source. Note that this error is largely dominated by the error on the strong coupling. Adopting a more optimistic attitude ${ }^{17}$ and assuming $\alpha_{s}=0.1184 \pm 0.0007$ evidently leads to a reduction down to $2.5 \%$.

Similar considerations apply to the analysis of the bottom quark mass but are outside the scope of this presentation.

\section{Direct Production of $\chi_{c 1}$ and $\chi_{c 2}$ at Electron-Positron colliders}

\subsection{Search for $\chi_{c 1}$ in the scanning mode}

With the forementioned high luminosity operation of BEPC in the energy region between 2 and $4.6 \mathrm{GeV}$ and plans to perform a dedicated energy scan, the possibility of direct resonant production of $\chi_{c 1}$ and $\chi_{c 2}$ through the reaction

$$
e^{+} e^{-} \rightarrow \chi_{c J}
$$

with $J=1$ or 2 is within reach. This reaction, originally suggested in Ref. 18 long time ago, proceeds through two virtual photons. Its strength is characterized by the respective electronic widths, $\Gamma\left(\chi_{c J} \rightarrow e^{+} e^{-}\right) \equiv \Gamma_{J}$, which were investigated in Ref. 18. (At that time this investigation was motivated by the idea ${ }^{19}$ that direct resonant production of a $J^{P C}=1^{++}$quarkonium state, composed of bottom or top quarks, could proceed through the axial part of the neutral current, a possibility 
which seems to be out of reach.) The peak cross section for a narrow resonance, normalized to the point cross section $\sigma_{0}$, is given by

$$
R_{\text {peak }} \equiv \frac{\sigma_{\text {peak }}}{\sigma_{0}}=(2 J+1) \frac{3 \pi}{2 \alpha^{2} \sqrt{2 \pi}} \frac{\Gamma_{J}}{\Delta M} C_{\text {rad }} .
$$

For the present discussion we assume a Gaussian spread cms energy spread of the center-of-mass energy with width $\Delta M=1.5 \mathrm{MeV}$. The factor $C_{\mathrm{rad}} \approx(2 \Delta M / M)^{t} \approx$ 0.5 with $t=2 \frac{\alpha}{\pi}\left(\ln \left(s / m_{e}^{2}\right)-1\right)$ arises from radiative corrections due to initial-state radiation. In numerical form

$$
R_{\text {peak }}=(2 J+1) 0.0176 \frac{\Gamma_{J}[\mathrm{eV}]}{\Delta M[\mathrm{MeV}]} .
$$

Estimates for the electronic widths $\Gamma_{J}$ can be found in Table 2 of Ref. 18. A sizable model dependence has been observed with results for $J=1$ varying between $0.044 \mathrm{eV}$, as nearly rigorous lower limit and $0.46 \mathrm{eV}$ as optimistic choice. The results for $J=2$ are even more uncertain and vary between $0.0023 \mathrm{eV}$ and $0.027 \mathrm{eV}$. Helicity conservation leads to $\Gamma_{J}=0$ for $J=0$ up to terms of order $\left(m_{e} / M_{\chi}\right)^{2}$. Let us, for the moment, concentrate on $J=1$ and use $\Gamma_{1}=0.4 \mathrm{eV}$ for the analysis and $1.5 \mathrm{MeV}$ for the energy spread. In this case we find

$$
R_{\text {peak }}(J=1) \approx 0.014
$$

for the peak cross section on top of a continuum cross section of $R_{\text {cont }} \approx 2$. This would require the sensitivity to a variation of the cross section to exceed a relative precision of 7 permille.

As an alternative one may concentrate on a selected final state with significantly improved signal-to-background ratio, which arises from the decay chain

$$
e^{+} e^{-} \rightarrow \chi_{J} \rightarrow J / \psi \gamma
$$

With a branching ratio $\operatorname{Br}\left(\chi_{c 1} \rightarrow J / \psi \gamma\right)=0.348 \pm 0.015$ this would lead to

$$
R_{\text {peak }}\left(e^{+} e^{-} \rightarrow \chi_{c 1} \rightarrow J / \psi \gamma\right) \approx 0.0049 .
$$

In this case the continuum background originates from the (non-resonant) radiative return to the $J / \psi$, which proceeds through initial state radiation. This cross section is given by $\left(z \equiv M_{J / \psi}^{2} / s\right)$

$$
\begin{aligned}
R_{B} & =\frac{9}{\alpha} \frac{\Gamma\left(J / \psi \rightarrow e^{+} e^{-}\right)}{M_{J / \psi}}(1-z)\left[\frac{1+z^{2}}{(1-z)^{2}} \ln \frac{1+\Delta}{1-\Delta}-\Delta\right] \\
& \approx \frac{9 \Gamma\left(J / \psi \rightarrow e^{+} e^{-}\right)}{\alpha\left(M_{\chi}-M_{J / \psi}\right)} \ln \frac{1+\Delta}{1-\Delta}
\end{aligned}
$$

with $z \equiv M_{J / \psi}^{2} / s$ and $\Delta \equiv \cos \theta_{\max }$ defining the acceptance range for the radiated photon. With $\theta_{\max }=30^{\circ}$ one finds $R_{B}=0.045$, to be contrasted with a resonance peak value $R_{\text {peak }}=0.0049$. 
We thus arrive at a signal/background ratio of roughly 0.10 and a relatively clean final state, namely a photon plus a lepton pair with an invariant mass of the $J / \psi$. To finally estimate the required event rates we consider the $e^{+} e^{-}$and $\mu^{+} \mu^{-}$decay modes of $J / \psi$ with branching ratios of $6 \%$ each. This affects signal and background in the same way with a reduction factor of 0.12 . The final $R$ value for the background reaction $e^{+} e^{-} \rightarrow J / \psi \gamma$ including the the branching ratio of $J / \psi$ into $e^{+} e^{-}$or $\mu^{+} \mu^{-}$amounts to 0.0054 and corresponds to 38 picobarn. To observe a one-sigma effect at one point would require 100 events, corresponding to $2.6 \mathrm{pb}^{-1}$ per point. More realistically, with 3 points and $24 \mathrm{pb}^{-1}$ per point (corresponding to $3 \sigma$ ) one would definitely be on the safe side. (Note that these estimates are based on the optimistic assumption of $\Gamma_{e}=0.4 \mathrm{eV}$.)

On the other hand, $24 \mathrm{pb}^{-1}$ correspond to about 0.3 million hadronic events, which would give a statistical precision of better than $3.3 \times 10^{-3}$ in the total cross section, to be compared to the variation of $7 \times 10^{-3}$. The total cross section measurement thus could lead to an independent confirmation of the resonance.

A more refined analysis ${ }^{20}$ will exploit the difference in the angular distribution of photon and leptons from signal and background, as well as the non-vanishing relative phase of signal versus background, which could give rise to an interesting interference pattern.

\subsection{Search for $\chi_{c 1}$ through the radiative return}

As an alternative one might consider using the radiative return (including small angle photon emission) from one fixed energy point where a large amount of luminosity has been collected. For a case study let us take $E_{\mathrm{cm}}=3.770 \mathrm{GeV}$. The $R$-value for the production of a narrow resonance with mass $M$ and electronic width $\Gamma_{e}$ plus a photon emitted at arbitrary angle is given by

$$
R=\frac{9}{\alpha} \frac{\Gamma_{e}}{M} \frac{1+z^{2}}{(1-z)}(L-1)
$$

For $M=3.511 \mathrm{GeV}$ and $\Gamma_{e}=0.4 \mathrm{eV}$ as before one finds $R_{\chi \gamma}=7.8 \times 10^{-6}$, corresponding to $\sigma_{\chi \gamma}=190 \mathrm{fb}^{-1}$. Assuming, furthermore, an integrated luminosity of $3 \mathrm{fb}^{-1}$, one arrives at 23 events in the $\gamma \gamma \mu^{+} \mu^{-}$or $e^{+} e^{-}$configuration where the invariant mass of the lepton pair plus one of the photons is given by the mass of the $\chi_{1}$ state. It remains to be seen if the energy resolution is sufficient to distinguish this signal from the non-resonant background $e^{+} e^{-} \rightarrow \gamma \gamma J / \psi \rightarrow \gamma \gamma \mu^{+} \mu^{-}$or $e^{+} e^{-}$.

\section{Acknowledgments}

The author would like to thank H. Czyż and A. Denig for helpful discussions.

\section{References}

1. For an overview see: Liang Yan, these proceedings. 
2. for an overview see: Thomas Mehen, these proceedings.

3. J. H. Kühn and M. Steinhauser, Nucl. Phys. B 619, 588 (2001) [Erratum-ibid. B 640, 415 (2002)] [hep-ph/0109084].

4. J. H. Kühn, M. Steinhauser and C. Sturm, Nucl. Phys. B 778, 192 (2007) [hepph/0702103 [HEP-PH]].

5. K. G. Chetyrkin, J. H. Kühn, A. Maier, P. Maierhofer, P. Marquard, M. Steinhauser and C. Sturm, Phys. Rev. D 80, 074010 (2009) [arXiv:0907.2110 [hep-ph]].

6. K. Chetyrkin, J. H. Kühn, A. Maier, P. Maierhofer, P. Marquard, M. Steinhauser and C. Sturm, Theor. Math. Phys. 170, 217 (2012) [arXiv:1010.6157 [hep-ph]].

7. K. G. Chetyrkin, J. H. Kühn and M. Steinhauser, Phys. Lett. B 371, 93 (1996) [hep$\mathrm{ph} / 9511430]$.

8. K. G. Chetyrkin, J. H. Kühn and M. Steinhauser, Nucl. Phys. B 482, 213 (1996) [hep-ph/9606230].

9. K. G. Chetyrkin, J. H. Kühn and C. Sturm, Eur. Phys. J. C 48, 107 (2006) [hep$\mathrm{ph} / 0604234]$.

10. R. Boughezal, M. Czakon and T. Schutzmeier, Phys. Rev. D 74, 074006 (2006) [hep$\mathrm{ph} / 0605023]$.

11. A. Maier, P. Maierhofer and P. Marquard, Phys. Lett. B 669, 88 (2008) [arXiv:0806.3405 [hep-ph]].

12. A. Maier, P. Maierhofer, P. Marquard and A. V. Smirnov, Nucl. Phys. B 824, 1 (2010) [arXiv:0907.2117 [hep-ph]]

13. C. Sturm, JHEP 0809, 075 (2008) [arXiv:0805.3358 [hep-ph]].

14. Evgenii Baldin, private communication.

15. Achim Denig, private communication.

16. S. Bethke, A. H. Hoang, S. Kluth, J. Schieck, I. W. Stewart, S. Aoki, M. Beneke and S. Bethke et al., "Workshop on Precision Measurements of $\alpha_{s}$," arXiv:1110.0016 [hep-ph].

17. J. Beringer et al. [Particle Data Group Collaboration], Phys. Rev. D 86 (2012) 010001.

18. J. H. Kühn, J. Kaplan and E. G. O. Safiani, "Electromagnetic Annihilation of e+ eInto Quarkonium States with Even Charge Conjugation," Nucl. Phys. B 157 (1979) 125.

19. J. Kaplan and J. H. Kühn, "Direct Production of ${ }^{++}$States in $e^{+} e^{-}$Annihilation,", Phys. Lett. B 78 (1978) 252.

20. H. Czyż, J. H. Kühn, in preparation. 